\title{
Association Between Amyloid Accumulation and Sleep in Patients With Idiopathic REM Sleep Behavior Disorder
}

\begin{abstract}
Hanul Lee ${ }^{1}$, Hyunjin Cho ${ }^{1}$, Yeong Sim Choe ${ }^{1,2}$, Sang Won Seo ${ }^{1 *}$ and Eun Yeon Joo ${ }^{1 *}$
'Department of Neurology, Neuroscience Center, School of Medicine, Samsung Medical Center, Sungkyunkwan University, Seoul, South Korea, ${ }^{2}$ Department of Health Sciences and Technology, Samsung Advanced Institute for Health Sciences \& Technology (SAIHST), Sungkyunkwan University, Seoul, South Korea
\end{abstract}

Background and Objectives: Amyloid-beta protein may lead to sleep disturbance and eventually develop cognitive impairment. Idiopathic rapid eye movement (REM) sleep behavior disorder (iRBD) is a predictor of neurodegeneration, yet there have been limited studies evaluating the relationship between cognitive decline and amyloid accumulation in iRBD patients. The aim of this study is to investigate the clinical and sleep characteristics of iRBD patients and its association with amyloid deposition.

Edited by:

Milena Pavlova

Brigham and Women's Hospital and Harvard Medical School, United States

Reviewed by:

Anna Szucs,

Semmelweis University, Hungary Lyudmila S. Korostovtseva, Almazov National Medical Research Centre, Russia

*Correspondence: Eun Yeon Joo ejoo@skku.edu. eunyeon.joo@gmail.com Sang Won Seo sw72.seo@samsung.com sangwonseo@empas.com

Specialty section:

This article was submitted to Sleep Disorders,

a section of the journal

Frontiers in Neurology

Received: 31 March 2020 Accepted: 03 November 2020 Published: 03 December 2020

Citation:

Lee H, Cho H, Choe YS, Seo SW and Joo EY (2020) Association Between Amyloid Accumulation and Sleep in Patients With Idiopathic REM Sleep Behavior Disorder.

Front. Neurol. 11:547288 doi: 10.3389/fneur.2020.547288
Methods: We enroll 23 iRBD patients (mean age, 65.8 years; male, 73.9\%), and their mean history of clinically suspected RBD was 6.5 years. All underwent 18F-flutemetamol amyloid PET completed polysomnography (PSG) and questionnaires. Patients were classified into two groups according to amyloid deposition as amyloid positive and negative. Clinical and sleep parameters were compared between groups and were correlated with amyloid deposition, calculated as a standardized uptake value ratio (SUVR).

Results: Four patients (17.4\%) were revealed to be amyloid positive, and they showed increased percentage of wake after sleep onset (WASO), stage N1, and stage N2 sleep and worse on the Stroop Word Color Test compared to amyloid negative patients. Global SUVR was correlated with total sleep time, sleep efficiency, WASO, and N1 sleep, and these sleep parameters were associated with a part of default mode network of brains such as orbitofrontal, dorsolateral pre-frontal, and left temporal areas.

Conclusion: iRBD patients with amyloid deposition have worse sleep quality than patients without amyloid. Relationship between fragmented sleep and amyloid deposition in the default mode network may be crucial to elucidate the disease progress of RRB.

\section{Keywords: idiopathic REM sleep behavior disorder, amyloid, sleep, cognition, default mode network}

\section{INTRODUCTION}

Rapid eye movement (REM) sleep behavior disorder (RBD) is a well-known parasomnia characterized by absence of muscle atonia during REM sleep. RBD presents with dream enacting motor behaviors or vocalization during REM sleep, such as sleep talking, screaming, punching, or falling off the bed (1). These behaviors are associated with violent contents of the dream during REM sleep (2), which may eventually result in physical injury to patients or bedside partners. 
The term idiopathic RBD (iRBD) is used in the absence of other known causes for secondary RBD, which are mainly neurodegenerative diseases associated with synucleinopathy, such as dementia with Lewy bodies, Parkinson's disease, or multiple system atrophy $(3,4)$. The association of iRBD with synucleinopathy is well-established $(5,6)$. Evidence has increased regarding phenoconversion of iRBD into synucleinopathies presenting with parkinsonism or dementia $(5,7,8)$. The relationship between $\mathrm{iRBD}$ and synucleinopathy corresponds with the progression of disease pathology affecting brain anatomical structures $(9,10)$.

Numerous data showed that iRBD patients perform poorly on neuropsychological tests (11-16), and mild cognitive impairment (MCI) is frequently observed in iRBD patients $(14,17,18)$. However, results were inconsistent across studies depending on which cognitive domain was impaired, population heterogeneity, small sample size, and the use of different cognitive tasks with variable sensitivity (19).

Amyloid-beta protein is known as one of the key molecules in pathophysiology of Alzheimer's disease (AD), which is the most common cause of dementia in the elderly and characterized by memory deficit and cognitive dysfunction (20). The primary cause for the disease is not completely known; thus, mixed proteinopathy of amyloid and tau is the proposed pathology and results in amyloid plaque and neurofibrillary tangle deposition (21). Amyloid beta may be increased with the normal aging process and show positivity among the cognitively normal elderly. The deposition of amyloid would result in cognitive decline in episodic memory, visuospatial function, and global cognition (22). Recent neuroimaging techniques such as amyloid positron emission tomography (PET) represents a potential major advance in the assessment of subjects with cognitive impairment by binding fluorescent isotopes in fibrillary beta amyloid in the brain tissues (23).

Amyloid deposition, sleep, and cognitive function are closely interrelated. AD patients show substantial alteration in sleep structure and quality due to amyloid deposition (24). This change develops during the early stage of disease before the cognitive symptom appears (25). Furthermore, the sleep disturbance increases cerebral amyloid beta level and subsequently elevates the risk of $\mathrm{AD}$ (26). RBD symptoms are not uncommon in $\mathrm{AD}$ patients $(27,28)$, and cognitive decline is commonly observed in iRBD patients (11-16). The relationship between amyloid deposition and $\mathrm{RBD}$ symptoms and the role of brain amyloid in the disease course of $\mathrm{iRBD}$ have not been clarified yet. In the present study, we hypothesize that amyloid accumulation contributes to sleep disturbance and eventually cognitive impairment in iRBD patients and aims to investigate its relationships with sleep and cognition in those patients.

\section{MATERIALS AND METHODS}

\section{Study Participants}

A total of 32 treatment-naïve iRBD patients who consecutively visited the Samsung Medical Center sleep clinic between June 2017 and January 2019 were enrolled in the present study. The diagnosis of iRBD is based on the International Classification of Sleep Disorders 3rd edition (ICSD-3) criteria (29).

All participants provided written informed consent and the study protocol was approved by the Ethics Committee of Samsung Medical Center. Patients who reported typical RBD motor or vocalization symptoms were eligible for the study. Two individual neurologists assessed patients to determine cognitive dysfunction or parkinsonism during the initial enrollment. Five patients with a history of cancer, three with a history of minor stroke, and one with parkinsonian features were excluded. Finally, 23 patients completed questionnaires including registration survey, RBD questionnaire Korean version (RBDQ-KR), Epworth Sleepiness Scale (ESS), Beck Depression Inventory (BDI), Geriatric Depression Scale (GDS-K), Hospital Anxiety and Depression Scale (HADS), Pittsburgh Sleep Quality Index (PSQI), and the Scales for Outcomes in Parkinson's disease-Autonomic (SCOPA-AUT). Registration survey contained questions about alcohol, caffeine consumption, habitual sleep time, habitual sleep latency, underlying disease, and onset of RBD symptom. In particular, alcohol consumption was assessed using questions about average weekly consumption during the past 12 months (30). All participants fulfilled sleep diary more than 2 weeks before enrollment. Sleep latency and sleep duration were calculated from the data.

Patients' cognitive function and parkinsonism were evaluated using the Korean version of the Mini-Mental State Examination (K-MMSE), Clinical Dementia Rating scale (CDR), Korean version of Montreal Cognitive Assessment (K-MOCA), and Unified Parkinson's Disease Rating Scale (MDS-UPDRS). After enrollment, all patients underwent overnight polysomnography (PSG), neuropsychological test, brain MRI, and 18F-flutemetamol PET scan.

\section{Sleep Study (Overnight Polysomnography)}

Polysomnography (PSG) was recorded with standard electrodes and sensors using Embla N7000 (Medcare Flaga, Iceland). The study was performed using the standard PSG protocol with C3-A2, C4-A1, F3-A2, F4-A1, O3-A2, and O2-A1 electroencephalography electrodes, four electrooculography electrodes, chin, and both anterior tibialis electromyogram, and electrocardiography sensors were monitored. Thoracic and abdominal movement were monitored using two plethysmography belts, nasal and oral airflow was measured using a nasal pressure transducer, and oxygen saturation was monitored using a thermistor and pulse oximetry. Synchronized video recording was used to monitor abnormal sleep behaviors or vocalization during sleep. Sleep parameters such as total sleep time (TST), sleep latency, waking after sleep onset (WASO), sleep efficiency (SE), sleep stages (N1, N2, N3, REM sleep, \%), arousals (arousal index, respiratory-related arousal, spontaneous arousal, movement-related arousal), and the apnea-hypopnea index (AHI) were collected. Sleep stages and sleep events scoring were defined according to American Academy of Sleep Medicine (AASM) manual for sleep and associated events (31). 


\section{Neuropsychological Test}

All patients completed a standardized neuropsychological battery, the Seoul Neuropsychological Screening Battery (SNSB), to evaluate cognitive function (32). One neuropsychological specialist had done every patient's test. Test was done by face-toface examination, including interviewing the patient's caregivers. The battery is composed of multiple tests to assess attention and executive function domains such as information processing, verbal fluency, verbal memory, visual memory, and language. The score of the individual test was computed as $z$-score and averaged as composite score for each domain.

\section{Brain MRI Scanning}

Participants underwent a 3D volumetric brain MRI scan. An Achieva 3.0 T MRI scanner (Philips; Best, the Netherlands) was used to acquire 3D T1 turbo field echo MRI data. Imaging parameters were as follows: sagittal slice thickness of $1.0 \mathrm{~mm}$ with $50 \%$ overlap, no gap, repetition time of $9.9 \mathrm{~ms}$, echo time of $4.6 \mathrm{~ms}$, flip angle of $8^{\circ}$, and matrix size of $240 \times 240$ pixels reconstructed to $480 \times 480$ pixels over a $240-\mathrm{mm}$ field of view.

\section{F-Flutemetamol Amyloid PET Scanning}

Flutemetamol PET scans were conducted with a Discovery 600 PET/CT scanner (GE), Discovery 690 PET/CT scanner (GE), Discovery STE PET/CT scanner (GE), Biography MCT PET/CT scanner (Siemens), or Gemini TF PET/CT scanner (Philips) (33). The participants underwent a 20 -min PET scan $(4 \times$ $5 \mathrm{~min}$ dynamic frames) starting at $90 \mathrm{~min}$ after intravenous injection of $185 \mathrm{MBq} \pm 10 \%$ of $18 \mathrm{~F}$-flutemetamol. Low-dose CT was utilized for attenuation correction before scans. Visual interpretation of 18F-flutemetamol PET images relied upon a systematic review of five brain regions (frontal, parietal, posterior cingulate and precuneus, striatum, and lateral temporal lobes). If any of the brain regions systematically reviewed for 18Fflutemetamol PET was positive in either hemisphere, the scan was considered positive.

Flutemetamol PET images were co-registered to individual MRIs, which were normalized to a T1-weighted MRI template. The quantitative regional values of flutemetamol retention on the spatially normalized flutemetamol images were obtained using an automated volume of interest (VOI) analysis with the automated anatomical labeling atlas. Data processing was performed using the Statistical Parametric Mapping (SPM) program, Version 8 in the Matlab 2014b (Mathworks, Natick, MA, United States). A total of 28 cortical VOIs were selected from each hemisphere using the AAL atlas. Regional cerebral standardized uptake value ratios (SUVRs) of flutemetamol were calculated by dividing each cortical VOI's SUV by the mean SUV of the pons value (a reference). The cerebral cortical VOIs chosen for this study consisted of frontal areas (superior and middle frontal gyri, the medial part of the superior frontal gyri, the opercular and triangular parts of the inferior frontal gyri, the supplementary motor areas, the orbital part of the superior, middle, and inferior orbital frontal gyri, the rectal gyri, and the olfactory cortices), posterior cingulate gyri, parietal areas (superior and inferior parietal areas, supramarginal and angular gyri, and precuneus), lateral temporal areas (superior, middle, and inferior temporal gyri and Heschl's gyri), and occipital areas (superior, middle, and inferior occipital gyri, cuneus, calcarine fissures, and lingual and fusiform gyri) bilaterally. A global flutemetamol uptake was calculated from the volume-weighted average SUVR of 28 cortical VOIs from each hemisphere.

TABLE 1 | Demographics and clinical information in patients with idiopathic RBD.

\begin{tabular}{|c|c|c|c|c|}
\hline & Total & Amyloid (+) & Amyloid (-) & $p$-value \\
\hline Age & $65.80(60.30-69.80)$ & 70.60 (59.18-74.53) & 65.50 (60.30-68.60) & 0.194 \\
\hline Disease onset age & $59.00(50.00-66.00)$ & $63.00(52.25-73.00)$ & $57.00(50.00-65.00)$ & 0.330 \\
\hline Sex, Male:Female & $17: 6$ & $4: 0$ & $13: 6$ & 0.539 \\
\hline Body mass index, $\mathrm{kg} / \mathrm{m}^{2}$ & $24.90(23.40-26.70)$ & $25.70(24.72-28.17)$ & $24.90(23.25-26.40)$ & 0.282 \\
\hline Hypertension & 8 & 0 & 8 & 0.257 \\
\hline Caffeine, $n /$ day & $1.00(0.00-2.50)$ & $0.00(0.00-1.88)$ & $1.00(1.00-3.00)$ & 0.081 \\
\hline Habitual sleep time, h & $6.50(5.75-7.63)$ & $7.00(5.00-7.88)$ & $6.50(5.75-7.25)$ & 0.731 \\
\hline Habitual sleep latency, h & $0.28(0.10-0.50)$ & $0.50(0.20-0.88)$ & $0.25(0.10-0.50)$ & 0.161 \\
\hline Epworth sleepiness scale & $5.00(3.25-8.75)$ & $6.00(3.25-9.50)$ & $5.00(3.25-8.75)$ & 0.887 \\
\hline Pittsburgh sleep quality index & $3.00(2.00-4.00)$ & $4.00(3.00-4.00)$ & $3.00(2.00-3.25)$ & 0.147 \\
\hline K-MMSE & 28.00 (25.00-30.00) & $26.50(21.25-29.50)$ & 29.00 (27.00-30.00) & 0.299 \\
\hline Clinical dementia rating scale & $0.50(0.00-0.50)$ & $0.50(0.12-0.50)$ & $0.00(0.00-0.50)$ & 0.325 \\
\hline Beck depression inventory II & $12.00(9.00-17.00)$ & $7.50(5.00-7.88)$ & $16.00(9.00-20.00)$ & 0.056 \\
\hline
\end{tabular}

RBDQ-KR, REM sleep behavior disorder Questionnaire-Korean version; K-MMSE, Korean version of Mini-Mental Statue Examination. All variables are presented as median and interquartile range (Q1-Q3). ${ }^{*} p<0.05$, Mann-Whitney U-test. 


\section{Voxel-Wise Regression Analysis}

SPM analysis was performed without global count normalization because the flutemetamol PET images were changed to the uptake ratio (SUVR) parametric image using the pons region of interest (ROI) uptake value. Statistical comparisons between sleep parameters and SUVR were performed on a voxelby-voxel basis using linear regression with age as covariate. Statistically increased flutemetamol retention areas of the brain were investigated at a height threshold of false discovery ratecorrected $p<0.05$ with an extent threshold of 100 voxels. The MNI coordinates of the local maximum of each cluster were converted into Talairach coordinates.

\section{Statistical Analysis}

All continuous variables were analyzed using the independent $t$-test or Mann-Whitney $U$-test, and categorical variables were analyzed using Fisher's exact test. Correlation between clinical and PSG parameters and PET SUVR values were analyzed using Pearson correlation test or Spearman correlation. Age and education years were used as control variables for correlation analysis. Statistical analyses were performed with SPSS software (version 25.0, SPSS Inc., Chicago, IL, United States). A $p<0.05$ was considered to indicate statistical significance.

\section{RESULTS}

Out of 23 iRBD patients who completed full evaluations, 16 patients (69.5\%) were confirmed by typical RBD behaviors correlated with simultaneously occurring dream mentation as well as REM without atonia (RWA) on polysomnographic recording. The remaining seven patients demonstrated sufficient RWA although RBD behaviors did not exhibit on sleep study night. All patients had typical clinical history of RBD with dreamenacting behaviors. Clinically suspected RBD history was varied from 2 to 10 years (mean 6.5 years).

Four patients (17.4\%) were positive on 18F-flutemetamol PET. Demographics and clinical data were not different between amyloid-positive and amyloid-negative patients (Table 1).

In sleep data, the amyloid-positive group had significantly higher N1 sleep time $(p=0.014)$ and lower N2 sleep time ( $p=$ 0.027 ) than the amyloid-negative group (Table 2 ).

Amyloid-positive patients showed worse performance in the Stroop Word Test $(p=0.003)$ and Stroop Color Test $(p=$ 0.015; Table 3). Seven of 23 patients (30.4\%) were revealed to have MCI state, and two of them were amyloid-positive. The numbers of MCI were not different between amyloid-positive and amyloid-negative groups (50 vs. $35.71 \%$, Fisher's exact test, $p=0.557)$.

Correlation analyses between clinical data and imaging data were performed in 23 patients. A global amyloid burden based on SUVR was negatively correlated with TST (Pearson's coefficient $-0.626, p=0.003)$ and SE $(-0.627, p=0.002)$ and positively correlated with WASO $(0.582, p=0.006)$ and N1 sleep time $(0.728, p<0.001$; Figure 1). SVLT total score was also negatively correlated with RBDQ-KR $(-0.511, p=0.025)$. RBDQ-KR
TABLE 2 | Comparison of polysomnography parameters in in patients with idiopathic RBD according to amyloid deposition.

\begin{tabular}{|c|c|c|c|}
\hline & Amyloid (+) & Amyloid (-) & $p$-value \\
\hline Time in bed, min & 455.30 (375.90-516.63) & $461.00(403.75-479.00)$ & 0.929 \\
\hline $\begin{array}{l}\text { Total sleep time, } \\
\text { min }\end{array}$ & 256.25 (204.25-399.00) & 384.50 (320.13-438.50) & 0.148 \\
\hline Sleep latency, min & $9.00(3.25-11.38)$ & $7.00(4.25-17.13)$ & 0.733 \\
\hline $\begin{array}{l}\text { REM sleep latency, } \\
\text { min }\end{array}$ & $80.25(64.50-166.50)$ & 74.50 (57.13-96.38) & 0.610 \\
\hline Sleep efficiency, \% & 67.35 (42.70-83.23) & 89.00 (71.70-91.40) & 0.074 \\
\hline N1 sleep time, \% & 31.60 (18.60-41.08) & 15.45 (8.00-21.13) & $0.014^{*}$ \\
\hline N2 sleep time, \% & 51.05 (42.80-55.93) & 63.30 (54.53-67.53) & $0.027^{*}$ \\
\hline N3 sleep time, \% & $0.00(0.00-0.00)$ & 0.25 (0.00-1.38) & 0.063 \\
\hline REM sleep time, \% & $17.35(4.88-36.73)$ & 22.90 (14.83-26.63) & 0.551 \\
\hline WASO, min & 133.30 (59.00-272.40) & 43.40 (33.90-100.50) & 0.052 \\
\hline WASO, \% & 31.10 (14.68-56.23) & 10.00 (7.70-25.70) & 0.052 \\
\hline $\begin{array}{l}\text { Apnea-hypopnea } \\
\text { index, /h }\end{array}$ & $9.40(2.68-15.83)$ & $9.00(1.90-17.00)$ & 0.935 \\
\hline Arousal index, /h & 23.60 (16.30-24.53) & 14.45 (8.65-27.35) & 0.733 \\
\hline $\begin{array}{l}\text { Respiratory } \\
\text { arousal }\end{array}$ & $5.40(2.05-6.28)$ & $5.50(1.05-11.45)$ & 0.858 \\
\hline Snore arousal & $0.60(0.33-6.35)$ & $0.20(0.00-0.65)$ & 0.073 \\
\hline RERA & $4.40(1.78-7.70)$ & $1.60(0.02-2.75)$ & 0.065 \\
\hline $\begin{array}{l}\text { Spontaneous } \\
\text { arousal }\end{array}$ & $7.90(6.33-9.33)$ & 5.15 (2.28-9.65) & 0.394 \\
\hline $\begin{array}{l}\text { Movement } \\
\text { related arousal }\end{array}$ & $0.95(0.15-3.85)$ & $0.10(0.00-1.20)$ & 0.434 \\
\hline PLM index, $\mathrm{h}$ & 13.05 (1.25-48.33) & $5.40(0.00-28.30)$ & 0.709 \\
\hline
\end{tabular}

REM, Rapid eye movement; WASO, Wake after sleep onset; RERA, Respiratory effort related arousal; PLM, Periodic limb movement during sleep. All variables are presented as median and interquartile range (Q1-Q3). ${ }^{*} p<0.05$, Mann-Whitney U-test.

showed a tendency to have negative correlation with verbal memory composite score $(p=0.064)$.

Voxel-wise regression analysis was performed with four parameters (TST, SE, WASO, and N1 sleep time), which were significant in the global SUVR correlation analyses and showed relevant associations with cortical retention of flutemetamol in bilateral orbitofrontal, dorsolateral pre-frontal, and left lateral temporal areas $(p<0.05$, False discovery rate corrected; Figure 2).

\section{DISCUSSION}

In the present study, amyloid deposit was hypothetically responsible for sleep disturbance and cognitive impairment in iRBD patients and the relationships between amyloid burden and sleep and cognitive data of patients were investigated. Four patients (17.4\%) were amyloid-positive on PET scan and their sleep quality was worse than in amyloid-negative patients. Several cognitive domains were more impaired in amyloid-positive patients, but the percentage of patients with MCI state was not higher than amyloid-negative patients.

Considering the median age of study participants (63.3 years), amyloid-positive rate $(17.4 \%)$ in iRBD patients was comparable 
TABLE 3 | Comparison of neuropsychological data in patients with idiopathic RBD according to amyloid deposition.

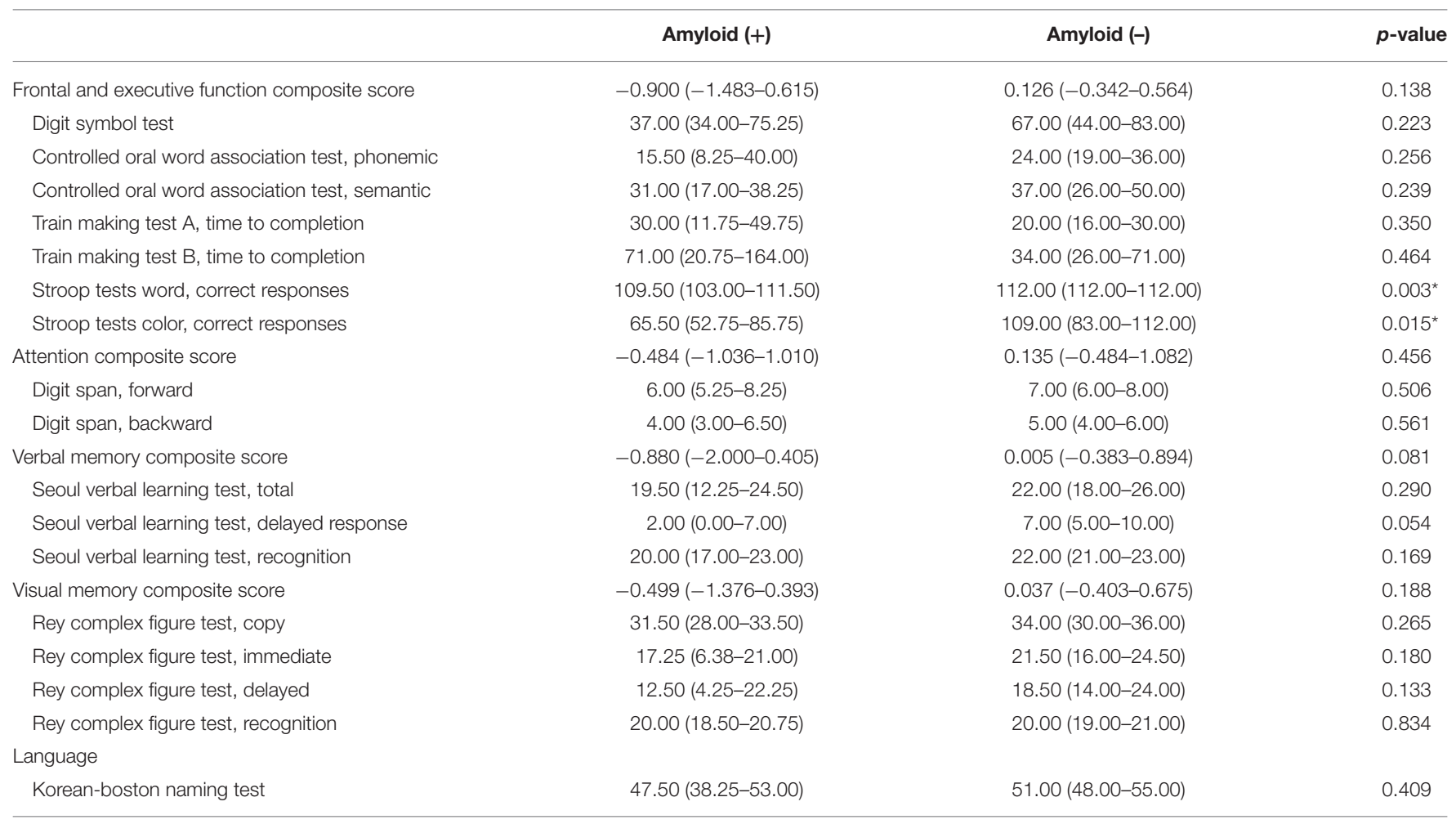

All variables are presented as median and interquartile range (Q1-Q3). ${ }^{*} p<0.05$, Mann-Whitney U-test.

to that of cognitively normal elderly in a population-based study (18.3\%) (34). Literature showed that RBD symptoms were manifested in $10 \%$ of $\mathrm{AD}$ patients $(27,28)$ and in $1.06 \%$ of the cognitively normal elderly population (35). RBD is regarded as the prodromal feature of synucleidopathy diseases $(5,7,8,27$, $36,37)$, whereas it is accompanied with $\mathrm{AD}$ patients in relatively small portion $(10 \%)(27,28)$. Cognitive decline is commonly observed in iRBD patients and prevalence of MCI in RBD patient is up to $35-50 \%(14,38)$. This is the first study to investigate the positivity rate of amyloidopathy in $\mathrm{iRBD}$ patients and the association between RBD and other neurodegenerative diseases.

iRBD patients in this study also reported overall good sleep quality (PSQI and ISI) and daytime function (ESS and SSS), which in line with previous reports $(1,39)$. Subjective cognitive function was also well-preserved (K-MMSE and CDRS). In spite of the fact that amyloid-group differences of the above parameters were not significant, objective sleep parameters of amyloid-positive patients presented more fragmented sleep than amyloid-negative patients. Amyloid-positive iRBD patients had smaller N1 and larger N2 sleep \%. Sleep efficiency was much decreased in the amyloid-positive group (64.43\%, normal $\geq$ $85 \%)$, but statistically indifferent with amyloid negative patients (81.6\%, $p=0.074)$. WASO $\%$ also showed a tendency to be increased in amyloid-positive patients $(34.00 \%$ in amyloid positive vs. $16.76 \%$ in amyloid negative, $p=0.052)$. The small number of amyloid-positive patients $(n=4)$ may limit the statistical significance. From the clinical point of view, however, reduced sleep efficiency and increased WASO (more than 1/3 of total sleep) of amyloid-positive iRBD patients were enough to be interpreted as poor, fragmented sleep.

Intriguingly, sleep latency and AHI were within normal range considering ages in both groups and arousal index and periodic limb movement index were increased. It indicates that amyloid-positive iRBD patients have more fragmented sleep and worse sleep quality regardless of sleep-related breathing disorder, periodic limb movement disorder (PLMD), or sleep onset insomnia, and it enlightens the role of brain amyloid in development of sleep disturbances independent of specific sleep disorders in iRBD patients. With cognitive decline, the sleep becomes worse and shorter REM sleep and N3 sleep time and reduced sleep efficiency were found in AD patients (40). Taken together with our data, it is presumable that amyloid deposition in the brain may worsen the sleep efficiency in iRBD patients as well as cognitive-normal people (25).

Regarding neuropsychological testing, several frontal and executive function domains were worse in amyloid-positive patients. Four patients were revealed to be MCI state but dementia was not found. MCI state rate was not different according to amyloid deposition. More severe RBD symptoms scored by RBDQ-KR were significantly associated with impaired memory in iRBD patients. Previous reports showed that RBD patients had cognitive impairment in specific domains, such as attention, executive function, verbal and non-verbal memory, and visuospatial abilities (11-15). In particular, the Stroop Color Word Test and Trail Making Test are known to predict the development of dementia in iRBD patients (41). Participants 

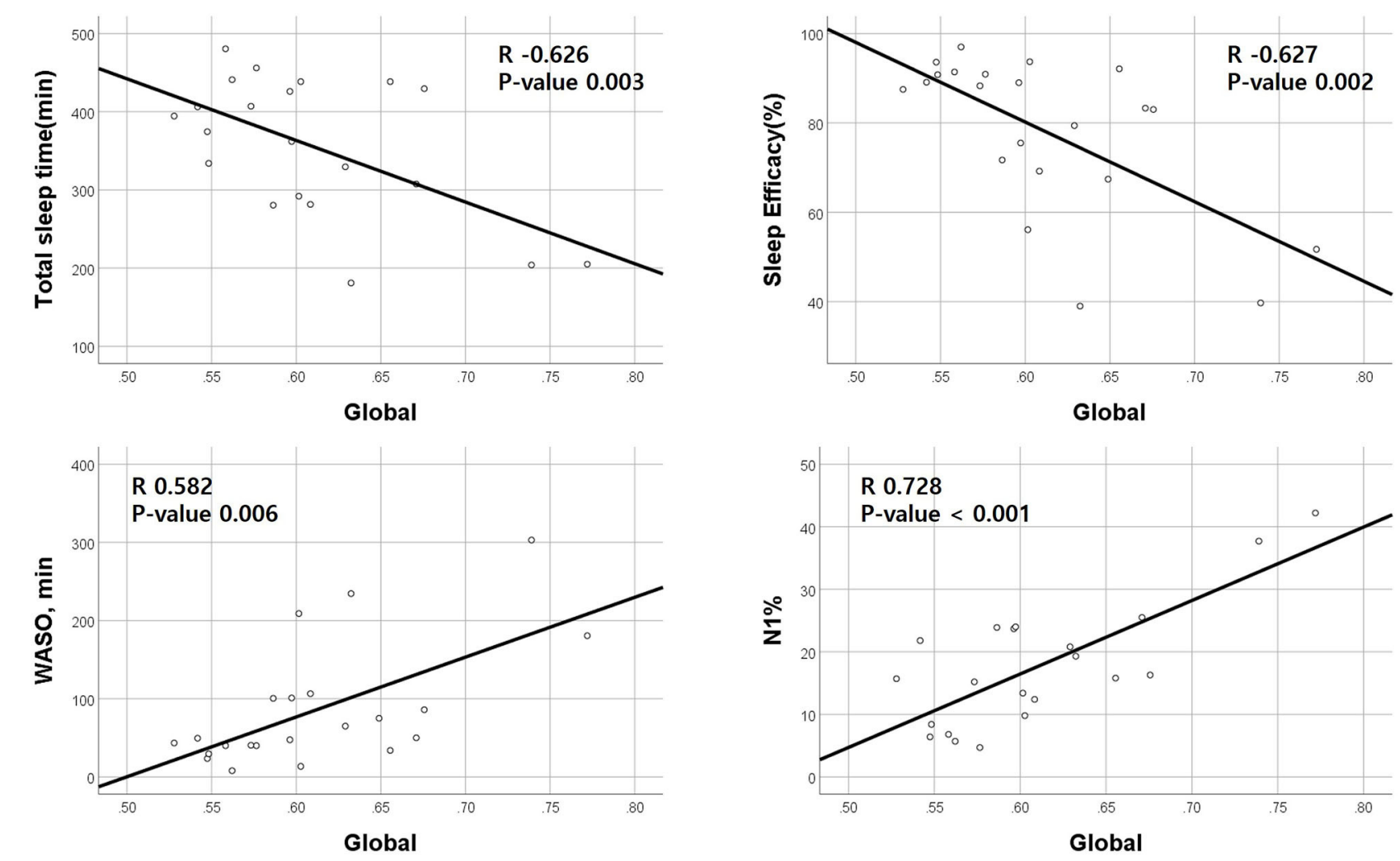

FIGURE 1 | Correlations between positron emission tomography standardized uptake value ratio (PET SUVR) and polysomnography parameters. Amyloid deposition (SUVR) is negatively correlated with total sleep time and sleep efficiency and positively correlated with wakefulness after sleep parameters (WASO) and N1 sleep \%. The $X$-axis represents the amount of global amyloid deposition.

in this study did not report subjective cognitive impairment. Notably, amyloid-negative patients did not show lower scores in any neuropsychological domains than amyloid-positive patients. MCI occurs in $35-50 \%$ of iRBD patients (14) and a similar rate was observed in this study (30.4\% of 23 ). However, MCI prevalence was not different between amyloid-positive and amyloid-negative groups. It is uncertain whether amyloid burden is associated with the development of MCI based on these data.

Brain regions with high neuronal activity are considered more susceptible to amyloid deposition. The default mode network consists of precuneus, posterior cingulate, medial pre-frontal cortex, lateral temporal cortex, hippocampal formation, angular gyrus, and retrosplenial cortex, and it is regarded as the most vulnerable region to amyloid burden (42). A previous report in non-demented late middle-aged adults showed that sleep disturbances estimated using the ESS and Medical Outcomes Study Sleep Scale were associated with amyloid burden in default mode network (angular gyrus, frontal medial orbital cortex, cingulate gyrus, and precuneus) (43). A survey of communitydwelling older adults found that short sleep duration was associated with amyloid deposition in the brain (44).

iRBD patients in this study report good sleep quality (PSQI) and do not complain daytime sleepiness or insomnia related to sleep disturbances. They sleep enough (average $6.48 \mathrm{~h}$ ) and have regular sleep-wake pattern. However, a sleep study showed that patients had lower sleep efficiency and, in particular, amyloid-positive patients have more fragmented sleep. Essential parameters (TST, sleep efficiency, WASO, and N1 sleep time) for sleep integrity were significantly correlated with amyloid deposition in the part of default of network as well as global amyloid burden.

Since this is a cross-sectional study, it does not clarify the causal relationships among sleep disturbance, cognitive decline, and amyloid deposition in iRBD patients. We observed in this study that amyloid-positive patients showed more poor sleep quality and worse performance in some parts of neuropsychological tests. Two out of four amyloid-positive patients were revealed to have MCI state. Although the numbers of MCI were not different between amyloid-positive and amyloid-negative groups, it is presumable that amyloid deposition may lead to sleep disturbance and then cognitive decline in $\mathrm{iRBD}$ patients.

The present study had several limitations. First, the study included a small number of iRBD patients that have weakened the statistical power and overestimated the results. Second, amyloid-positive healthy controls without RBD symptoms were not included to evaluate the effects of amyloidopathy on iRBD. Third, the technical limitation of 


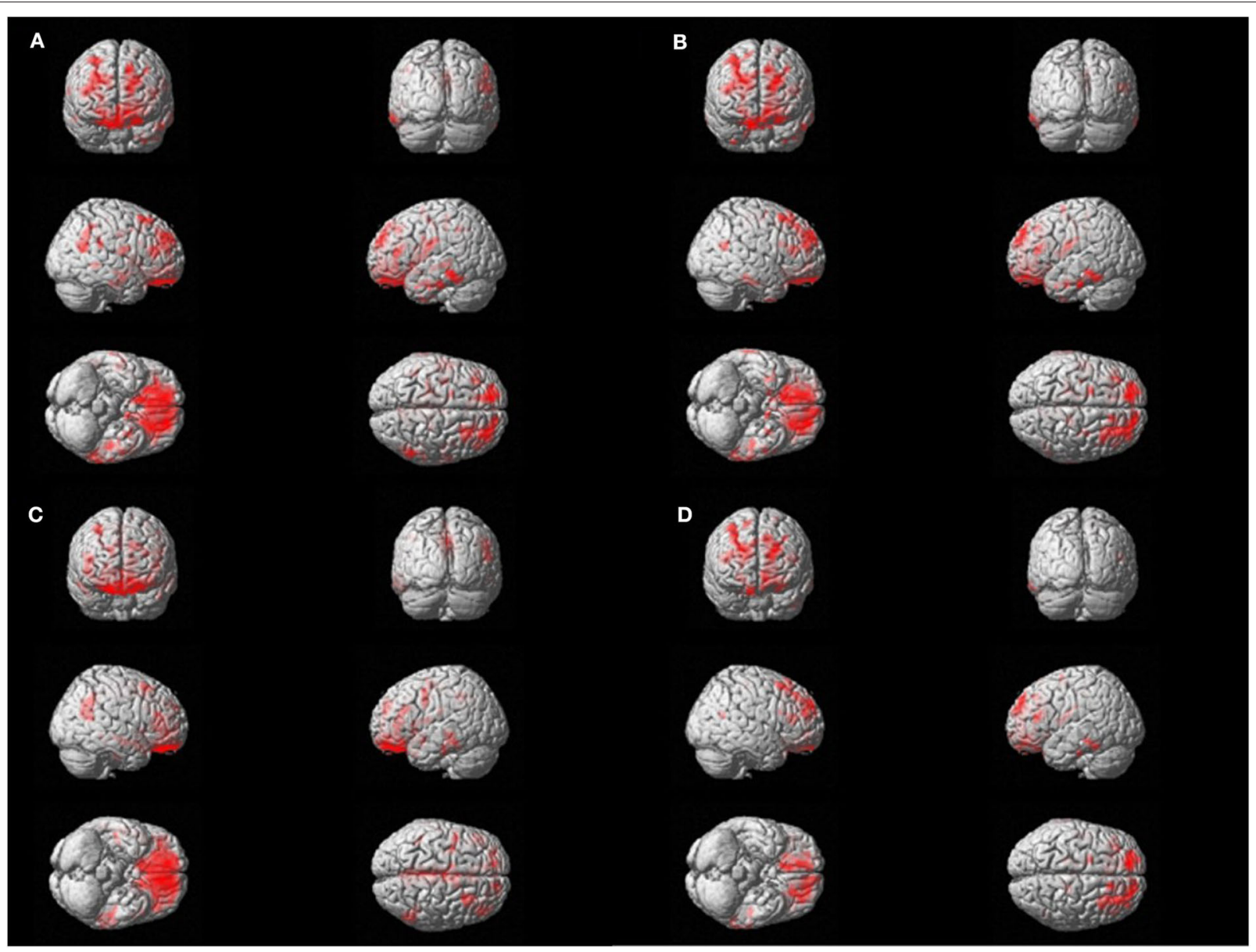

FIGURE 2 | Voxel-wise regression analysis between amyloid uptake and polysomnography parameters. Significant correlations are found with amyloid positron emission tomography standardized uptake value ratio (PET SUVR) and the sleep parameters in bilateral orbitofrontal, dorsolateral prefrontal, and left lateral temporal areas. The red area represents the three-dimensional location of areas that has correlation with each sleep parameter. FDR correction was done ( $p<0.05)$. (A) Total Sleep Time, min. (B) Sleep efficiency, \%. (C) Wakefulness after sleep onset, min. (D) N1 time, \%.

amyloid PET scan may hinder the interpretation of the results. Brainstem structures associated with RBD (45) are too small to evaluate with current PET technology (46). Despite the limitations, it has the strength that it demonstrates that the amyloid-positive iRBD patients have more disturbed sleep and more cognitive impairment compared with amyloidnegative patients for the first time and it suggests the possibility that early detection and intervention of sleep disturbance may prevent or delay the conversion to dementia in iRBD patients.

\section{DATA AVAILABILITY STATEMENT}

The raw data supporting the conclusions of this article will be made available by the authors, without undue reservation.

\section{ETHICS STATEMENT}

The studies involving human participants were reviewed and approved by Ethics Committee of Samsung Medical Center. The patients/participants provided their written informed consent to participate in this study.

\section{AUTHOR CONTRIBUTIONS}

HL, SS, and EJ contributed to conception and design of the study. $\mathrm{HL}, \mathrm{HC}$, and YC organized the database. YC contributed to image processing and figure making. HL wrote the first draft of the manuscript. All authors contributed to manuscript revision and read and approved the submitted version.

\section{FUNDING}

This research was supported by Samsung Medical Center Grant \#OTC1190671.

\section{ACKNOWLEDGMENTS}

This research was presented by poster on SLEEP 2019, titled as The relationship between amyloid accumulation and poor sleep in patients with idiopathic REM sleep behavior disorder. 


\section{REFERENCES}

1. Schenck $\mathrm{CH}$, Mahowald MW. REM sleep behavior disorder: clinical, developmental, and neuroscience perspectives 16 years after its formal identification in SLEEP. Sleep. (2002) 25:120-38. doi: 10.1093/sleep/ 25.2.120

2. Fantini ML, Corona A, Clerici S, Ferini-Strambi L. Aggressive dream content without daytime aggressiveness in REM sleep behavior disorder. Neurology. (2005) 65:1010-5. doi: 10.1212/01.wnl.0000179346.39655.e0

3. Fantini ML, Ferini-Strambi L, Montplaisir J. Idiopathic REM sleep behavior disorder: toward a better nosologic definition. Neurology. (2005) 64:780-6. doi: 10.1212/01.WNL.0000152878.79429.00

4. Jiang H, Huang J, Shen Y, Guo S, Wang L, Han C, et al. RBD and neurodegenerative diseases. Mol Neurobiol. (2017) 54:2997-3006. doi: 10.1007/s12035-016-9831-4

5. Postuma RB, Iranzo A, Hu M, Hogl B, Boeve BF, Manni R, et al. Risk and predictors of dementia and parkinsonism in idiopathic REM sleep behaviour disorder: a multicentre study. Brain. (2019) 142:74459. doi: 10.1093/brain/awz030

6. Postuma RB, Gagnon JF, Vendette M, Fantini ML, Massicotte-Marquez J, Montplaisir J. Quantifying the risk of neurodegenerative disease in idiopathic REM sleep behavior disorder. Neurology. (2009) 72:1296-300. doi: 10.1212/01.wnl.0000340980.19702.6e

7. Yao C, Fereshtehnejad SM, Dawson BK, Pelletier A, Gan-Or Z, Gagnon JF, et al. Longstanding disease-free survival in idiopathic REM sleep behavior disorder: Is neurodegeneration inevitable? Parkinsonism Relat Disord. (2018) 54:99-102. doi: 10.1016/j.parkreldis.2018.04.010

8. Postuma RB, Iranzo A, Hogl B, Arnulf I, Ferini-Strambi L, Manni R, et al. Risk factors for neurodegeneration in idiopathic rapid eye movement sleep behavior disorder: a multicenter study. Ann Neurol. (2015) 77:8309. doi: 10.1002/ana.24385

9. Lai YY, Siegel JM. Physiological and anatomical link between Parkinson-like disease and REM sleep behavior disorder. Mol Neurobiol. (2003) 27:13752. doi: 10.1385/MN:27:2:137

10. Braak H, Del Tredici K, Rub U, de Vos RA, Jansen Steur EN, Braak E. Staging of brain pathology related to sporadic Parkinson's disease. Neurobiol Aging. (2003) 24:197-211. doi: 10.1016/S0197-4580(02)00065-9

11. Ferini-Strambi L, Di Gioia MR, Castronovo V, Oldani A, Zucconi M, Cappa SF. Neuropsychological assessment in idiopathic REM sleep behavior disorder (RBD): does the idiopathic form of RBD really exist? Neurology. (2004) 62:41-5. doi: 10.1212/01.WNL.0000101726.69701.FA

12. Massicotte-Marquez J, Decary A, Gagnon JF, Vendette M, Mathieu A, Postuma RB, et al. Executive dysfunction and memory impairment in idiopathic REM sleep behavior disorder. Neurology. (2008) 70:1250-7. doi: 10.1212/01.wnl.0000286943.79593.a6

13. Terzaghi M, Sinforiani E, Zucchella C, Zambrelli E, Pasotti C, Rustioni $\mathrm{V}$, et al. Cognitive performance in REM sleep behaviour disorder: a possible early marker of neurodegenerative disease? Sleep Med. (2008) 9:34351. doi: 10.1016/j.sleep.2007.06.013

14. Gagnon JF, Vendette M, Postuma RB, Desjardins C, Massicotte-Marquez J, Panisset $\mathrm{M}$, et al. Mild cognitive impairment in rapid eye movement sleep behavior disorder and Parkinson's disease. Ann Neurol. (2009) 66:3947. doi: 10.1002/ana.21680

15. Marques A, Dujardin K, Boucart M, Pins D, Delliaux M, Defebvre L, et al. REM sleep behaviour disorder and visuoperceptive dysfunction: a disorder of the ventral visual stream? J Neurol. (2010) 257:38391. doi: 10.1007/s00415-009-5328-7

16. Fantini ML, Farini E, Ortelli P, Zucconi M, Manconi M, Cappa S, et al. Longitudinal study of cognitive function in idiopathic REM sleep behavior disorder. Sleep. (2011) 34:619-25. doi: 10.1093/sleep/34.5.619

17. Iranzo A, Molinuevo JL, Santamaria J, Serradell M, Marti MJ, Valldeoriola F, et al. Rapid-eye-movement sleep behaviour disorder as an early marker for a neurodegenerative disorder: a descriptive study. Lancet Neurol. (2006) 5:572-7. doi: 10.1016/S1474-4422(06)70476-8

18. Molano J, Boeve B, Ferman T, Smith G, Parisi J, Dickson D, et al. Mild cognitive impairment associated with limbic and neocortical lewy body disease: a clinicopathological study. Brain. (2010) 133 (Pt. 2):54056. doi: 10.1093/brain/awp280
19. Gagnon JF, Bertrand JA, Genier Marchand D. Cognition in rapid eye movement sleep behavior disorder. Front Neurol. (2012) 3:82. doi: $10.3389 /$ fneur.2012.00082

20. Fratiglioni L, Grut M, Forsell Y, Viitanen M, Grafstrom M, Holmen K, et al. Prevalence of Alzheimer's disease and other dementias in an elderly urban population: relationship with age, sex, and education. Neurology. (1991) 41:1886-92. doi: 10.1212/WNL.41.12.1886

21. Lane CA, Hardy J, Schott JM. Alzheimer's disease. Eur J Neurol. (2018) 25:59-70. doi: 10.1111/ene.13439

22. Baker JE, Lim YY, Pietrzak RH, Hassenstab J, Snyder PJ, Masters CL, et al. Cognitive impairment and decline in cognitively normal older adults with high amyloid-beta: a meta-analysis. Alzheimer's Dementia. (2017) 6:10821. doi: 10.1016/j.dadm.2016.09.002

23. Fantoni ER, Chalkidou A, Brien JTO, Farrar G, Hammers A. A systematic review and aggregated analysis on the impact of amyloid PET brain imaging on the diagnosis, diagnostic confidence, and management of patients being evaluated for Alzheimer's disease. J Alzheimers Dis. (2018) 63:78396. doi: 10.3233/JAD-171093

24. Lucey BP, Bateman RJ. Amyloid-beta diurnal pattern: possible role of sleep in Alzheimer's disease pathogenesis. Neurobiol Aging. (2014) 35 (Suppl. 2):S2934. doi: 10.1016/j.neurobiolaging.2014.03.035

25. Ju YE, McLeland JS, Toedebusch CD, Xiong C, Fagan AM, Duntley SP, et al. Sleep quality and preclinical Alzheimer disease. JAMA Neurol. (2013) 70:587-93. doi: 10.1001/jamaneurol.2013.2334

26. Ooms S, Overeem S, Besse K, Rikkert MO, Verbeek M, Claassen JA. Effect of 1 night of total sleep deprivation on cerebrospinal fluid beta-amyloid 42 in healthy middle-aged men: a randomized clinical trial. JAMA Neurol. (2014) 71:971-7. doi: 10.1001/jamaneurol.2014.1173

27. Boeve BF, Silber MH, Parisi JE, Dickson DW, Ferman TJ, Benarroch EE, et al. Synucleinopathy pathology and REM sleep behavior disorder plus dementia or parkinsonism. Neurology. (2003) 61:40-5. doi: 10.1212/01.WNL.0000073619.94467.B0

28. Ferman TJ, Boeve BF, Smith GE, Lin SC, Silber MH, Pedraza O, et al. Inclusion of RBD improves the diagnostic classification of dementia with Lewy bodies. Neurology. (2011) 77:875-82. doi: 10.1212/WNL.0b013e31822c9148

29. American Academy of Sleep M. International classification of sleep disorders. Diagnost Coding Manu. (2005) 51-5. doi: 10.1007/978-1-4939-6578-6_27

30. Choi SJ, Lee SI, Joo EY. Habitual alcohol consumption and metabolic syndrome in patients with sleep disordered breathing. PLOS ONE. (2016) 11:e0161276. doi: 10.1371/journal.pone.0161276

31. Berry RB, Brooks R, Gamaldo C, Harding SM, Lloyd RM, Quan SF, et al. AASM Scoring Manual Updates for 2017 (Version 2.4). J Clin Sleep Med. (2017) 13:665-6. doi: 10.5664/jcsm.6576

32. Ahn HJ, Chin J, Park A, Lee BH, Suh MK, Seo SW, et al. Seoul Neuropsychological Screening Battery-dementia version (SNSB-D): a useful tool for assessing and monitoring cognitive impairments in dementia patients. J Korean Med Sci. (2010) 25:1071-6. doi: 10.3346/jkms.2010.25.7.1071

33. Kim SE, Lee B, Park S, Cho SH, Kim SJ, Kim Y, et al. Clinical significance of focal ss-amyloid deposition measured by (18)F-flutemetamol PET. Alzheimers Res Ther. (2020) 12:6. doi: 10.1186/s13195-019-0577-x

34. Roberts RO, Aakre JA, Kremers WK, Vassilaki M, Knopman DS, Mielke MM, et al. Prevalence and outcomes of amyloid positivity among persons without dementia in a longitudinal, population-based setting. JAMA Neurol. (2018) 75:970-9. doi: 10.1001/jamaneurol.2018.0629

35. Haba-Rubio J, Frauscher B, Marques-Vidal P, Toriel J, Tobback N, Andries D, et al. Prevalence and determinants of rapid eye movement sleep behavior disorder in the general population. Sleep. (2018) 41:zx197. doi: 10.1093/sleep/zsx197

36. Claassen DO, Josephs KA, Ahlskog JE, Silber MH, Tippmann-Peikert $\mathrm{M}$, Boeve BF. REM sleep behavior disorder preceding other aspects of synucleinopathies by up to half a century. Neurology. (2010) 75:4949. doi: 10.1212/WNL.0b013e3181ec7fac

37. You S, Jeon SM, Cho YW. Rapid eye movement sleep behavior disorder. $J$ Sleep Med. (2018) 15:1-7. doi: 10.13078/jsm. 18001

38. Terzaghi M, Zucchella C, Rustioni V, Sinforiani E, Manni R. Cognitive performances and mild cognitive impairment in idiopathic rapid eye movement sleep behavior disorder: results of a longitudinal follow-up study. Sleep. (2013) 36:1527-32. doi: 10.5665/sleep.3050 
39. Fernandez-Arcos A, Iranzo A, Serradell M, Gaig C, Santamaria J. The clinical phenotype of idiopathic rapid eye movement sleep behavior disorder at presentation: a study in 203 consecutive patients. Sleep. (2016) 39:12132. doi: 10.5665/sleep.5332

40. Peter-Derex L, Yammine P, Bastuji H, Croisile B. Sleep and Alzheimer's disease. Sleep Med Rev. (2015) 19:29-38. doi: 10.1016/j.smrv.2014.03.007

41. Genier Marchand D, Montplaisir J, Postuma RB, Rahayel S, Gagnon JF. Detecting the cognitive prodrome of dementia with lewy bodies: a prospective study of REM sleep behavior disorder. Sleep. (2017) 40. doi: 10.1093/sleep/zsw014

42. Bero AW, Yan P, Roh JH, Cirrito JR, Stewart FR, Raichle ME, et al. Neuronal activity regulates the regional vulnerability to amyloid-beta deposition. Nat Neurosci. (2011) 14:750-6. doi: 10.1038/nn.2801

43. Sprecher KE, Bendlin BB, Racine AM, Okonkwo OC, Christian BT, Koscik RL, et al. Amyloid burden is associated with self-reported sleep in nondemented late middle-aged adults. Neurobiol Aging. (2015) 36:2568-76. doi: 10.1016/j.neurobiolaging.2015.05.004

44. Spira AP, Gamaldo AA, An Y, Wu MN, Simonsick EM, Bilgel M, et al. Selfreported sleep and beta-amyloid deposition in community-dwelling older adults. JAMA Neurol. (2013) 70:1537-43. doi: 10.1001/jamaneurol.2013.4258

45. Hogl B, Stefani A, Videnovic A. Idiopathic REM sleep behaviour disorder and neurodegeneration - an update. Nat Rev Neurol. (2018) 14:4055. doi: 10.1038/nrneurol.2017.157

46. Braun DJ, Van Eldik LJ. In vivo brainstem imaging in alzheimer's disease: potential for biomarker development. Front Aging Neurosci. (2018) 10:266. doi: 10.3389/fnagi.2018.00266

Conflict of Interest: The authors declare that the research was conducted in the absence of any commercial or financial relationships that could be construed as a potential conflict of interest.

Copyright (c) 2020 Lee, Cho, Choe, Seo and Joo. This is an open-access article distributed under the terms of the Creative Commons Attribution License (CC BY). The use, distribution or reproduction in other forums is permitted, provided the original author(s) and the copyright owner(s) are credited and that the original publication in this journal is cited, in accordance with accepted academic practice. No use, distribution or reproduction is permitted which does not comply with these terms. 